\title{
COMENTARIO A «ALGUNAS CONFUSIONES ACERCA DE LOS PROBLEMAS MORALES DE LA DIVERSIDAD CULTURAL» DE ERNESTO GARZÓN VALDÉS
}

\author{
José María Sauca \\ Universidad Carlos III de Madrid
}

\begin{abstract}
RESUMEN. Se reformulan los errores denunciados por Ernesto Garzón Valdés en torno al tratamiento ético de la diversidad cultural mediante nueve tesis que son comentadas con intención moderadamente crítica.
\end{abstract}

Palabras clave: Ernesto Garzón Valdés, diversidad cultural.

ABSTRACT. Misconceptions about the ethical treatment of cultural diversity, as highlighted by Ernesto Garzón Valdés, are here reformulated into nine theses which are commented in a soft-critical way.

Keywords: Ernesto Garzón Valdés, cultural diversity. 
1 tema de esta nota se ocupa del tratamiento teórico que Ernesto GARZÓN VALDÉs ha desarrollado sobre la cuestión de las dimensiones morales del pluralismo cultural. Esta cuestión ha venido recibiendo su atención de una manera continuada durante los últimos quince años y, en mi opinión, ha devenido un espacio de intersección de algunas de las tesis centrales de su obra tales como la fundamentación de la moral; la delimitación del ámbito de la tolerancia; la justificación del paternalismo; los límites del coto vedado; la defensa de la democracia representativa; cuestiones todas ellas planteadas con un espíritu polemista cuyo escenario de fondo es la defensa de la autonomía individual de los seres humanos.

Tres han sido los contextos en los que ha desarrollado esta cuestión; a saber, en primer lugar, la evaluación moral de las tesis políticas y antropológicas formuladas sobre la cuestión indígena en América Latina y singularmente en México (GARZÓN VALDÉS, 1992a: 4-32; GARZÓN VALDÉS, 1993a: 37-62; GARZÓN VALDÉS, 1993b: 31-60 y GARZÓN VALDÉs, 1993c: 219-242). En segundo lugar, los problemas éticos imbricados en el pluralismo cultural; GARZÓN VALDÉS, 1997a: 10-23; GARZÓN VALDÉS, 1997b: 59-68; GARZÓN VALDÉS, 1999a: 21-33 y GARZÓN VALDÉS, 2004a: 93-115). En tercer lugar, la fundamentación de los derechos humanos en términos netamente universalistas (por todos: GARZÓN VALDÉS en MugUeRZA, 1998: 88-112; GARZÓN VALDÉS, 1999b: 85-111 y GARZÓN VALDÉS, 2004b: 15-35).

El trabajo específico del que me voy a ocupar se titula «Algunas confusiones acerca de los problemas morales de la diversidad cultural» y fue publicado en 1996 en el libro homenaje al filósofo mexicano Fernando SALMERÓN (GARZÓN VALDÉS, 1996: 81109). Se incluiría en el segundo apartado de la clasificación tripartita señalada en el párrafo precedente y se caracteriza por denunciar las confusiones teóricas que sufren los presupuestos éticos del multiculturalismo. Son cinco los errores básicos: confusión entre tolerancia y relativismo moral; confusión entre diversidad cultural y enriquecimiento moral; confusión entre identidad personal y promoción de la comunidad de origen; confusión entre unidad cultural y unidad institucional y, finalmente, confusión entre derechos jurídicos y derechos morales. A partir de estos errores, se infieren una pluralidad de equivocaciones que GARZÓN VALDÉS denomina «errores derivados» y que completan la comprensión de las insuficiencias morales del multiculturalismo. En lo que sigue, agruparé argumentos pertenecientes a ambas categorías de errores, reformulándolos en torno a nueve tesis que comento brevemente.

Tesis 1: La diferencia cultural no implica la inconmensurabilidad de culturas.

En términos generales, considero complicado establecer un conjunto de parámetros evaluatorios que permitan determinar la preferencia de una u otra cultura en un juicio unitario y unívoco. La mesurabilidad es una cualidad de difícil verificación cuando se predica de conjuntos complejos de variables interrelacionadas. El carácter provisional de las conclusiones ofrecidas por el uso de indicadores de desarrollo socio-económico, de calidad de vida o de evaluación del grado de satisfacción de los miembros de cada una de las diferentes sociedades, resulta mucho más fidedigno que los indicios utilizables para la evaluación de una cultura. En cualquier caso, aun cuando pudieran aplicarse a éstas los criterios aplicables a las sociedades o, siquiera algunos de ellos, no resultarían relevantes para la determinación de su mayor o menor dignidad como 
corrobora de manera pacífica la antropología contemporánea. Más singularmente, esta argumentación se fortalece cuando focalizamos nuestro interés en la dimensión moral de las diferentes culturas. Si consideramos, por un lado, que ninguna cultura, en la medida en que como suele decir el propio GARZÓn VALDÉS en la estela de HART, es una cultura suicida que representa el mal moral absoluto y, por otro, que ninguna cultura existente ha concretado la utopía de hacer coincidir su moralidad positiva con una moral esclarecida, parece plausible comprender que todas ellas suponen construcciones colectivas de relevancia moral. Finalmente, a este dato se ha de añadir que todas las culturas poseen un carácter internamente plural y que son realidades dinámicas susceptibles de cambios evolutivos. Tomando en consideración estas ideas, no parece viable sostener que pueda procederse a un juicio unívoco comparativo sobre la evaluación de la calidad moral de cada realidad cultural y es mucho más plausible sostener que cada una de ellas supone plurales discursos de construcción de la moralidad.

Tesis 2: No hay relación conceptual entre tolerancia y relativismo.

GARZÓN VALDÉS distingue entre intolerancia, tolerancia boba o insensata y tolerancia sensata. Se incurriría en la primera en aquellos casos en que se prohíbe una conducta sin justificación suficiente para ello. En la segunda cuando se transige con comportamientos cuya prohibición se halla justificada moralmente. Se celebraría la tercera cuando se permite lo que no está justificado prohibir a lo que añade la característica de su estabilidad en el tiempo. Este planteamiento resume las tesis centrales del autor que han sido desarrolladas en otras sedes (significativamente, GARZÓN VALDÉs, 1992b: 1623 y GARZÓN VALDÉS, 1993d: 423-428) a las que me remito. En esta ocasión quisiera centrarme en dos cuestiones específicas que no fueron tratadas en las precedentes ocasiones. En primer lugar, estimo aceptable considerar que la tolerancia, a la manera de otro tipo de virtudes tales como la clemencia o la prudencia, presentan un ámbito de aplicación más adecuado en las comúnmente denominadas éticas de excelencia, frente a las éticas de deber (por todos FULLER, 1969: 5-6), dado que facilitan los espacios del consejo, la recomendación, la superación, etc. La relevancia aquí de esta reflexión obedece a que este tipo de éticas tienen una dependencia de modelos culturalmente definidos mucho más intensa que las éticas basadas en deberes que reportan un carácter prevalentemente formal. En segundo lugar, el relativismo moral no implica necesariamente el valor moral de la tolerancia salvo contradicción en los términos. Sin embargo, sí parece plausible sostener que hay una cierta consistencia pragmática de carácter meramente estratégico que anima a la adopción de esa actitud sobre la base, por un lado, de la imposibilidad de fundamentar un juicio moral de forma objetiva o intersubjetiva y, por otro, de la virtualidad racional a largo plazo de evitar o minimizar conflictos.

Tesis 3: No hay relación entre relativismo moral y pluralismo cultural.

GARZÓN VALDÉS señala plausiblemente que todo relativismo es en sí mismo una forma de etnocentrismo. Sin embargo, creo que procede apuntar tres modulaciones. En primer lugar, esta afirmación es consistente desde un punto de vista ético pero es discutible desde un punto de vista metaético porque una tesis ética relativista implica la aceptación de la posiblidad de otros puntos de vista morales que se hallen fundamentados de manera alternativa e incompatible. Desde esta perspectiva, el relativista moral podrá sostener tesis incompatibles con el pluralismo cultural basado en el etnocentris- 
mo de su particular sistema ético (de manera similar a las tesis del objetivismo moral) e impugnarlas. Sin embargo, no podrá metaéticamente hablando sostener la inaceptabilidad de otros sistemas morales o de las implicaciones morales de otros sistemas culturales. En segundo lugar, y sobre la base del plano ético, puede sostenerse, de modo análogo a como se argumentaba en la tesis anterior respecto a la tolerancia y el relativismo, que el tipo de etnocentrismo que delimita el relativismo moral es un etnocentrismo preferentemente abierto y falible que opera desde una perspectiva pragmática propiciando una estrategia tendente al reconocimiento del culturalmente diferente. En tercer lugar, el objetivismo moral en la medida en que sea compatible con la tolerancia, implica la determinación de un patrón de regularidad desde el que evaluar las excepciones. Trasladado al ámbito cultural, esta tesis es proclive a sostener el carácter de anormalidad moral de la tesis minoritaria, mientras que la tesis relativista tendería a una calificación uniforme de las plurales manifestaciones culturales sin recurrir a una prelación de preferencia.

\section{Tesis 4: La democracia no presupone el relativismo moral.}

GARZÓN VALDÉS ha argumentado en numerosas ocasiones en favor de la democracia y sobre la base de argumentos morales que pretenden alcanzar la consideración de objetivamente válidos. Sin embargo, en la medida en que la democracia se constituya como un procedimiento de toma de decisiones por mayorías, no puede aparecer como el procedimiento apto para adoptar decisiones morales que sólo son justificables por remisión a una fundamentación objetiva. El procedimiento volitivo que caracteriza a la democracia no puede operar como el procedimiento realista o cuasirealista de conocimiento de la moral. En esta lógica, las tesis objetivistas en materia ética tienden a establecer, precisamente, los límites y las excepciones al procedimiento democrático legítimo. Por el contrario, aquellas tesis favorables a la democracia que mantienen posiciones relativistas en materia moral, no pueden oponer a la democracia otra cosa diferente que más democracia. La vieja reflexión kelseniana sobre esta cuestión sigue teniendo vigencia: «en efecto, todos los grandes metafísicos se han decidido por la autocracia y contra la democracia; y los filósofos que han hablado la palabra de la democracia, se han inclinado casi siempre al relativismo empírico» (KELSEN, 1977: 154).

\section{Tesis 5: De la moral social no se sigue una moral justificada.}

Esta tesis me parece rotundamente acertada, por lo que no insistiré en su justificación. Por ello, cuando se pretende sostener una moral objetivamente justificada es necesario recurrir a una argumentación complementaria. La estrategia de GARZÓN VALDÉS en este intento consiste en recurrir a la idea de «base moral mínima» y de «imparcialidad». La primera se concreta en dos elementos: derecho a la autodefensa y prohibición de dañar arbitrariamente a sus semejantes. Por otro lado, la «exigencia de imparcialidad [se define como] el ser capaces de ponernos en la situación del otro, es también una invitación a la indulgencia y a la aceptación de actos que no calzan exactamente con alguno de nuestros propios sistemas normativos». Creo que tanto la determinación de semejanza como de alteridad, implican la remisión a elementos culturales de carácter sustantivo. Desde este punto de vista, el ámbito sobre el que van a desarrollarse el proyecto de una moral esclarecida es, necesariamente, el de la moral social con sus correspondientes componentes culturales e identitarios. De hecho, los recursos empleados en algunas ocasio- 
nes por GARZÓN VALDÉS como el de tender a una justificación humana de tintes empiristas o a la idea del mal en línea de DASGUPTA, supondrían un análisis de tipo cultural sobre el carácter cultural de las necesidades o de los males.

Tesis 6: La pertenencia a un grupo identitario particular no favorece el desarrollo de nuestra identidad.

GARZÓN VALDÉS entiende por grupo identitario particular aquel que está unido por sus propias memorias colectivas. Esta visión localista entendería que «todo intento de adoptar un ideal de vida cosmopolita, abierta a todas las tradiciones culturales, sin selección de origen étnico, frustraría u obstaculizaría la formación de una identidad personal plena. [Y añade] Sé por experiencia que esto es falso».

Admiro y comparto la atracción por la valoración cosmopolita, pero este cosmopolitismo no es un universalismo acultural sino que es una identidad cultural donde su narraciones y memorias colectivas de carácter abierto e híbrido aportan los mejores argumentos para su atractivo pero es, nuevamente, un particular cultural.

A partir de esta idea creo que son tres los argumentos que refuerzan la verosimilitud de la relevancia de estos relatos y memorias colectivas. En primer lugar, la idea de que estos relatos son condiciones de sentido de nuestros proyectos morales y suministran bienes primarios en términos rawlsianos. En segundo lugar, son la escuela de la moralidad; el espacio en el que aprendemos el proceso de construcción y reconstrucción de nuestras mores; y mediante ellas, de nuestra moralidad. Finalmente, porque son el soporte del bienestar individual. Como dice RAz: «la causa en defensa del multiculturalismo, $[. .$.$] su reclamo moral de respeto y de prosperidad se basa completamente$ en su importancia vital para la prosperidad de los seres humanos individuales. Esta causa es una defensa liberal, ya que pone énfasis en el papel de las culturas como presupuesto de la libertad individual y como un factor que le da forma y contenido. Dada la dependencia de la libertad individual y del bienestar con respecto a la calidad de miembro pleno de un grupo cultural respetado y próspero, no resulta sorprendente que el multiculturalismo emerja como un elemento central en cualquier programa político liberal decente para sociedades cuyos habitantes son miembros de una cantidad de grupos culturales viables» (RAZ, 1994: 193).

Tesis 7: El Derecho alternativo o no es relevante o no es moralmente aceptable.

GARZÓN VALDÉS insiste con criterio en la necesidad de justificar el ordenamiento jurídico. Sin embargo, no aprecio una diferencia relevante, a estos efectos, entre el derecho estatal y el Derecho alternativo pues las eventuales críticas que desde el punto de vista moral podrían formularse, son igualmente aplicables a ambas. En todo caso, a favor del segundo podrían alegarse los argumentos de tipo clásicamente liberal tendentes a favorecer la distribución del poder normativo. Las fórmulas de descentralización de la democracia representativa no se agotan en la distribución funcional o territorial del poder normativo sino también mediante fórmulas genéricamente aludidas por la expresión de pluralismo jurídico.

Tesis 8: No hay derechos morales de grupo.

Comparto la tesis de que grupos no tienen agencia moral y por tanto no son titulares de derechos morales. Desde esta perspectiva, no hay derechos morales colectivos. 
Sin embargo, creo que es plausible promover la aceptabilidad de la categoría de derechos morales de grupo. Estos derechos no son detentados por el grupo sino que corresponden a cada uno de los miembros individuales del mismo en tanto que miembros del colectivo y los tienen porque son derechos que se ejercitan concurrentemente. La cultura de un grupo puede entenderse, en este sentido, como un bien público no susceptible de distribuir los costes y beneficios de su existencia y que, necesariamente se disfruta de forma convergente. Los derechos culturales de los miembros de ese grupo serían derechos morales de cada uno de ellos para proteger ese interés público que existe en tanto se ejercita coincidentemente con otros.

Tesis 9: Los derechos de los individuos de las minorías culturales no aportan nada nuevo al efectivo disfrute de los derechos universales.

Es evidente que el efectivo disfrute de los derechos tradicionalmente incluidos en el ámbito del coto vedado reporta una posición moralmente plausible para todos los individuos. Sin embargo, hay una aportación relevante de las tesis favorables al pluralismo cultural que podría enunciarse como la aspiración a que los individuos puedan disfrutar de esos derechos sin dejar de ser miembros de su grupo cultural fundamentalmente, por la razón básica de que quieren seguir perteneciendo al mismo; esto es, porque quieren disfrutar razonablemente de esa situación que los demás individuos disfrutan sin tener que homogeneizarse culturalmente a un grupo dominante o, en su caso, modulando las exigencias que los denominados costes de transición cultural les podrían reportar.

Coda. Termino con una pequeña coda a una coda. El artículo que he comentado finaliza con un comentario de un «cordobés argentino» para un «cordobés mexicano» y reza: «Llega un momento en la vida en el que conviene dar gracias al destino cuando nos ha deparado el don de contar con amigos cabales. Siempre he dicho que mis amigos son las mejores personas del mundo y que ello es una muestra de la objetividad de los valores. Fernando SALMERÓN es un buen ejemplo de que estoy en lo cierto; no hay aquí relativismo que valga». Me permito cerrar estas notas suscribiendo las mismas palabras respecto de los maestros cabales [...] Ernesto GARZÓN VALDÉS es un buen ejemplo de que estoy en lo cierto; no hay aquí relativismo que valga.

\section{REFERENCIAS BIBLIOGRÁFICAS}

Fuller, Lon L., 1969: The Morality of Law, New Haven and London: Yale University Press. GARZÓN VALDÉS, E., 1992a: «Pluralidad étnica y unidad nacional. Consideraciones ético políticas sobre el caso de México», Iberoamericana, n. ${ }^{\circ}$ 16, pp. 4-32. (También en GARZÓN VALDÉs, E., 1993: Derecho, ética y política, Madrid: Centro de Estudios Constitucionales, pp. 911942).

- 1992b: «No pongas tus sucias manos sobre Mozart. Algunas consideraciones sobre el concepto de tolerancia», Claves de Razón Práctica, enero-febrero, n. ${ }^{\circ}$ 19, Madrid, pp. 16-23.

- 1993a: «El problema ético de las minorías étnicas», en Olivé, L. (comp.), 1993: Ética y diversidad cultural, México: Fondo de Cultura Económica (2. ${ }^{a}$ ed., idem, 2004), pp. 37-62. (También en Garzón Valdés, E., 1993: Derecho, ética y política, Madrid: Centro de Estudios Constitucionales, pp. 519-540). 
— 1993b: «El problema ético de las minorías étnicas», en BARRAGÁN, J., y RoDRíGueZ, A. (comps.): Las diversidades culturales en la formación y aplicación del Derecho», Caracas: Universidad Central de Venezuela, pp. 31-60.

— 1993c: «La antinomia entre las culturas», en GARZÓN VALDÉs, E., y SALMERÓN, F. (eds.): Epistemología y cultura. En torno a la obra de Luís Villoro, México: Universidad Nacional Autónoma de México, pp. 219-242.

— 1993d: «Algunas reflexiones más acerca del concepto de tolerancia. Comentarios a los comentarios de Pablo Navarro», Doxa. Cuadernos de Filosofía del Derecho, n. ${ }^{\circ} 14$, Alicante, pp. 423-428.

- 1996: «Algunas confusiones acerca de los problemas morales de la diversidad cultural», en Olivé, L., y Villoro, L. (eds.): Filosofía moral, educación e historia. Homenaje a Fernando Salmerón, México: Facultad de Filosofía y Letras, Instituto de Investigaciones Filosóficas, UNAM, pp. 81-109.

- 1997a: «Cinco confusiones acerca de la relevancia moral de la diversidad cultural», en Claves de la Razón Práctica, n. ${ }^{\circ}$ 74, pp. 10-23.

— 1997b: «Cinco confusiones acerca de la relevancia moral de la diversidad cultural», Este País, n. ${ }^{\circ} 73$, pp. $59-68$.

- 1999a: «A propósito del multiculturalismo», en Cuadernos Americanos, n. ${ }^{\circ}$ 73, pp. 21-33.

- 1999b: «Acerca de la universalidad de los derechos humanos y su posible fundamentación», en TORRES RIPA, J. (ed.): Derechos bumanos y diversidad cultural, Bilbao: Forum Deusto, Instituto de Derechos Humanos, Universidad de Deusto, pp. 85-111.

- 2004a: «Capítulo II: La pretendida relevancia moral de la diversidad cultural», Calamidades, Barcelona: Gedisa, pp. 93-135.

- 2004b: «Prólogo» a GonZÁlez Amuchastegui, J.: Autonomía, dignidad y ciudadanía. Una teoría de los derechos humanos, Valencia: Tirant lo Blanch, pp. 15-35.

Kelsen, H., 1977: Esencia y valor de la democracia, trad. LuengO, R., y LEGAZ, L., Barcelona: Labor.

MuguerzA, J., 1998: Ética, disenso y derechos humanos. En conversación con Ernesto Garzón Valdés, Madrid: Argés.

RAZ, J., 1994: «Multiculturalism: A Liberal Perspective», Dissent, winter, pp. 67-79. 